Sādhanā Vol. 39, Part 1, February 2014, pp. 149-164. (C) Indian Academy of Sciences

\title{
Kinetic mesh-free method for flutter prediction in turbomachines
}

\author{
$\mathrm{V}_{\text {RAMESH }}^{1, *}$ and S M DESHPANDE ${ }^{2}$ \\ ${ }^{1}$ Council for Scientific and Industrial Research, National Aerospace Laboratories, \\ Computational and Theoretical Fluid Dynamics Division, Bangalore 560 017, India \\ ${ }^{2}$ Engineering Mechanics Unit, Jawaharlal Nehru Centre for Advanced Scientific \\ Research, Jakkur, Bangalore 560 064, India \\ e-mail: vr@ctfd.cmmacs.ernet.in; smd@jncasr.ac.in
}

MS received 23 April 2013; accepted 29 July 2013

\begin{abstract}
The present paper deals with the development and application of a kinetic theory-based mesh-free method for unsteady flows. The method has the capability to compute on any arbitrary distribution of moving nodes. In general, computation of unsteady flow past multiple moving boundaries using conventional finite volume solvers are quite involved. They invariably require repeated grid generation or an efficient grid movement strategy. This approach becomes more difficult when there are many moving boundaries. In the present work, we propose a simple and an effective node movement strategy for the mesh-free solver. This can tackle the unsteady problems with moving boundaries in a much easier way. Using the present method we have computed unsteady flow in oscillating turbomachinery blades. A simple energy method has been used to predict flutter using the unsteady computations. The results compare well with the available experiments and other computations.
\end{abstract}

Keywords. Mesh-free kinetic upwind scheme; unsteady flows; modified CIR splitting; Eigenvector based weights; flutter prediction; turbomachinery blades.

\section{Introduction}

In this work, we present the development of a mesh-free method for flutter prediction in turbomachinery blades by computing unsteady flow past multiple oscillating blades. A code based on Least Squares Kinetic Upwind Method (LSKUM) (Ghosh \& Deshpande 1995) has been developed to tackle multiple oscillating bodies with moving nodes. This is an upwind code which uses Kinetic Flux Vector Splitting (KFVS). The present code is called TKFMG (Turbomachinery KFVS on MovinG nodes). LSKUM is a kinetic theory (Deshpande 1986) based mesh-free scheme for solving the inviscid compressible Euler equations of gas dynamics. This method has also been applied to compute viscous flows (Mahendra 2003). LSKUM has been extended to

*For correspondence 
applications with moving nodes (LSKUM_MN) (Ramesh 2001; Ramesh \& Deshpande 2001). In the present work, we use the Modified CIR splitting (MCIR) (Ramesh \& Deshpande 2004a,b, 2007) to obtain spatially higher order accuracy in LSKUM_MN. Essentially the dissipation term present in the first order scheme is suitably chosen to get an equivalent higher order scheme where the dissipation terms are comparable to the usual second order schemes. This leads to a single step higher order scheme without the need for a two level defect correction method (Ghosh \& Deshpande 1995), thereby reducing the computational costs. Apart from the implementation of MCIR in LSKUM_MN, we have also adopted the weighted least squares approach based on Eigenvector basis (Ramesh \& Deshpande 2007). In this approach the least squares approximations for all the derivatives reduce to an equivalent 1-D form. This again helps in further reducing the computational time. For the unsteady calculations, we have used the well-known dual time stepping procedure (Hong Luo et al 2001).

In order to validate the method for aeroelastic applications as well as demonstrate the power of the method to deal with multiple oscillating bodies we have computed the unsteady flow for the 4th standard aeroelastic test case (Bolcs \& Fransson 1986). Results from the present computations are also compared with other Euler computations (Gruber \& Cartsens 1998; Ji \& Liu 1999; Mani \& Feng 2001). All of them predict the flutter using energy method (Carta 1967). This is based on the net energy transfer from the airstream to the blade. In general, for turbomachinery blades the aerodynamic forces are much smaller compared to the inertial and stiffness forces. Thus, this allows one to do flutter predictions without the need for an active coupling between the CFD computations and a structural module. As such, the prediction of the unsteady flowfield for a given blade vibration mode is of essential importance. We have also used the same approach to predict the flutter.

\section{Least squares kinetic upwind method on moving nodes}

Here we briefly describe the formulation for 2-D LSKUM_MN. Consider the 2-D Boltzmann equation

$$
\frac{\partial f}{\partial t}+v_{1} \frac{\partial f}{\partial x}+v_{2} \frac{\partial f}{\partial y}=J,
$$

where $f$ is the velocity distribution function, $v_{1}$ and $v_{2}$ are the Cartesian components of the molecular velocity. $J$ represents a collision term which vanishes in the Euler limit, when $f$ is a Maxwellian distribution, $F$, which in two dimensions is given by;

$$
F=\frac{\rho}{I_{0}} \frac{\beta}{\pi} \exp \left[-\beta\left(v_{1}-u_{1}\right)^{2}-\beta\left(v_{2}-u_{2}\right)^{2}-I / I_{0}\right]
$$

where $\beta=1 /(2 R T), \rho$ is the fluid density, $I$ is internal energy variable, $I_{0}$ is the internal energy due to non-translational degrees of freedom, $I_{0}=(2-\gamma / \gamma-1) R T$ and $u_{1}$ and $u_{2}$ are the Cartesian components of the fluid velocity, $R$ is the gas constant and $T$ is the absolute temperature of the fluid. Therefore in the Euler limit it is enough to consider

$$
\frac{\partial F}{\partial t}+v_{1} \frac{\partial F}{\partial x}+v_{2} \frac{\partial F}{\partial y}=0
$$

Now let $w_{1}$ and $w_{2}$ represent the Cartesian components of the velocity of any moving node. 
In order to deal with problems involving moving nodes, we define the derivative of $F$ along the path of the node as:

$$
\left(\frac{d F}{d t}\right)_{m o v}=\left(\frac{\partial F}{\partial t}+w_{1} \frac{\partial F}{\partial x}+w_{2} \frac{\partial F}{\partial y}\right)
$$

Substituting for $(\partial F / \partial t)$ in Eq. (3) we get

$$
\left(\frac{d F}{d t}\right)_{m o v}+\left(v_{1}-w_{1}\right) \frac{\partial F}{\partial x}+\left(v_{2}-w_{2}\right) \frac{\partial F}{\partial y}=0 .
$$

Let $\overline{v_{1}}=v_{1}-w_{1}, \overline{v_{2}}=v_{2}-w_{2}$ be the components of the particle velocity relative to the moving node. Then Eq. (4) can be compactly written as

$$
\left(\frac{d F}{d t}\right)_{m o v}+\bar{v}_{1} \frac{\partial F}{\partial x}+\bar{v}_{2} \frac{\partial F}{\partial y}=0 .
$$

Using MCIR (Ramesh \& Deshpande 2004a,b) splitting, $\bar{v}_{1}$ and $\bar{v}_{2}$ are written as

$$
\bar{v}_{1}=\frac{\bar{v}_{1}+\left|\bar{v}_{1}\right| \phi_{1}}{2}+\frac{\bar{v}_{1}-\left|\bar{v}_{1}\right| \phi_{1}}{2}, \quad v_{2}=\frac{\bar{v}_{2}+\left|\bar{v}_{2}\right| \phi_{2}}{2}+\frac{\bar{v}_{2}-\left|\bar{v}_{2}\right| \phi_{2}}{2}
$$

where $\phi_{1}, \phi_{2}$ are dissipation control parameters corresponding to two components of molecular velocity. The dissipation control parameters are conveniently chosen as $\phi_{1}=\phi_{2}=\Delta r^{p}$, where $0<p<1$ and $\Delta r$ is the distance between a node and any point in its neighbourhood. We usually choose the closest point.

Using MCIR splitting for both the components of molecular velocity, the Boltzmann Eq. (5) can be written as

$$
\left(\frac{d F}{d t}\right)_{m o v}+\frac{\bar{v}_{1}+\left|\bar{v}_{1}\right| \phi_{1}}{2} \frac{\partial F}{\partial x}+\frac{\bar{v}_{1}-\left|\bar{v}_{1}\right| \phi_{1}}{2} \frac{\partial F}{\partial x}+\frac{\bar{v}_{2}+\left|\bar{v}_{2}\right| \phi_{2}}{2} \frac{\partial F}{\partial y}+\frac{\bar{v}_{2}-\left|\bar{v}_{2}\right| \phi_{2}}{2} \frac{\partial F}{\partial y}=0 .
$$

We define moment vector function $\Psi$ by

$$
\Psi=\left[1, v_{1}, v_{2}, I+\frac{v_{1}^{2}+v_{2}^{2}}{2}\right]^{T},
$$

and define the $\Psi$ moment as

$$
\langle\Psi, F\rangle \equiv \int_{0}^{\infty} d I \int_{-\infty}^{\infty} d v_{1} \int_{-\infty}^{\infty} d v_{2} \Psi F .
$$

Now the $\Psi$ moment of the Eq. (7) will lead to the modified moving kinetic flux vector split Euler equations

$$
\left(\frac{d U}{d t}\right)_{m o v}+\frac{\partial}{\partial x}\left(G X_{M}^{+}\right)+\frac{\partial}{\partial x}\left(G X_{M}^{-}\right)+\frac{\partial}{\partial y}\left(G Y_{M}^{+}\right)+\frac{\partial}{\partial y}\left(G Y_{M}^{-}\right)=0,
$$


where

$$
\begin{aligned}
\langle\Psi, F\rangle & \equiv \int_{0}^{\infty} d I \int_{-\infty}^{\infty} d v_{1} \int_{-\infty}^{\infty} d v_{2} \Psi F \equiv U \\
\left\langle\Psi, \frac{\bar{v}_{1}+\phi_{1}\left|\bar{v}_{1}\right|}{2} F\right\rangle & \equiv G X_{M}^{+} \\
\left\langle\Psi, \frac{\bar{v}_{1}-\phi_{1}\left|\bar{v}_{1}\right|}{2} F\right\rangle & \equiv G X_{M}^{-} \\
\left\langle\Psi, \frac{\bar{v}_{2}+\phi_{2}\left|\bar{v}_{2}\right|}{2} F\right\rangle & \equiv G Y_{M}^{+} \\
\left\langle\Psi, \frac{\bar{v}_{2}-\phi_{2}\left|\bar{v}_{2}\right|}{2} F\right\rangle & \equiv G Y_{M}^{-} .
\end{aligned}
$$

$U$ is the state vector given by $U=\left(\rho, \rho u_{1}, \rho u_{2}, \rho e\right)^{T}, e$ is the internal energy per unit mass given by $e=p / \rho(\gamma-1)+1 / 2\left(u_{1}^{2}+u_{2}^{2}\right), G X_{M}^{ \pm}$and $G Y_{M}^{ \pm}$are the modified split fluxes for the moving nodes. These modified moving split fluxes are expressed in terms of moving fluxes (Ramesh 2001; Ramesh \& Deshpande 2001) as

$$
\begin{aligned}
G X_{M}^{+} & =\frac{1}{2}\left\{\left(1+\phi_{1}\right) G X_{m}^{+}+\left(1-\phi_{1}\right) G X_{m}^{-}\right\}, \\
G X_{M}^{-} & =\frac{1}{2}\left\{\left(1+\phi_{1}\right) G X_{m}^{-}+\left(1-\phi_{1}\right) G X_{m}^{+}\right\}, \\
G Y_{M}^{+} & =\frac{1}{2}\left\{\left(1+\phi_{2}\right) G Y_{m}^{+}+\left(1-\phi_{2}\right) G Y_{m}^{-}\right\}, \\
G Y_{M}^{-} & =\frac{1}{2}\left\{\left(1+\phi_{2}\right) G Y_{m}^{-}+\left(1-\phi_{2}\right) G Y_{m}^{+}\right\} .
\end{aligned}
$$

Further, the moving split fluxes $G X_{m}^{ \pm}$and $G Y_{m}^{ \pm}$can be together expressed as

$$
G_{m}^{ \pm}=[A] G_{s}^{ \pm},
$$

where $G_{s}^{ \pm}$represent the split fluxes similar to that on static nodes. In this case the split fluxes are functions of velocity components relative to the node velocity. The matrix $[A]$ transforms the static fluxes to those on moving nodes. These fluxes for finite volume formulation have been given by Krishnamurthy et al (2004). The transformation matrix $[A]$ in terms of node velocities is given by

$$
[A]=\left[\begin{array}{cccc}
1 & 0 & 0 & 0 \\
w_{1} & 1 & 0 & 0 \\
w_{2} & 0 & 1 & 0 \\
\frac{w_{1}^{2}+w_{2}^{2}}{2} & w_{1} & w_{2} & 1
\end{array}\right] .
$$

The expressions for the split fluxes $G_{s}^{ \pm}$in terms of error functions and exponentials are given in Ramesh \& Deshpande (2007).

In order to develop an update scheme, we need to evaluate the space derivatives of various moving split fluxes. In LSKUM_MN the space derivatives are evaluated using least squares 
approximation. Consider any point $P_{o}$ as shown in figure 1. Assume that values of $F$ are available at $P_{o}$ and its immediate surrounding nodes, refered to as connectivity of point $P_{o}$. A first order approximation to the derivatives $\partial F / \partial x$ and $\partial F / \partial y$ using weighted least squares approach (Ghosh 1996) is then given by the following formulae

$$
\begin{aligned}
F_{x_{o}}^{(1)} & =\frac{\sum w_{i} \Delta y_{i}^{2} \sum w_{i} \Delta x_{i} \Delta F_{i}-\sum w_{i} \Delta x_{i} \Delta y_{i} \sum w_{i} \Delta y_{i} \Delta F_{i}}{\sum w_{i} \Delta x_{i}^{2} \sum w_{i} \Delta y_{i}^{2}-\left(\sum w_{i} \Delta x_{i} \Delta y_{i}\right)^{2}}, \\
F_{y_{o}}^{(1)} & =\frac{\sum w_{i} \Delta x_{i}^{2} \sum w_{i} \Delta y_{i} \Delta F_{i}-\sum w_{i} \Delta x_{i} \Delta y_{i} \sum w_{i} \Delta x_{i} \Delta F_{i}}{\sum w_{i} \Delta x_{i}^{2} \sum w_{i} \Delta y_{i}^{2}-\left(\sum w_{i} \Delta x_{i} \Delta y_{i}\right)^{2}},
\end{aligned}
$$

where, $\Delta x_{i}=x_{i}-x_{o}, \Delta y_{i}=y_{i}-y_{o}, \Delta F_{i}=F_{i}-F_{o}, w_{i}$ is a weight function and $\sum$ represents the summation over all the points in the neighbourhood (i.e., connectivity) of $P_{o}$.

The derivative at any point is a local property, hence in order to give more weightage to points closer to $P_{o}, w_{i}$ the weight function, is defined as a function of $d_{i}$, distance between any point $P_{i}$ and point $P_{o}$. The weight function considered in the present case is of the form $w_{i}=C_{i} / d_{i}^{2 n}$, where $n$ is an integer and $C_{i}$ is constant. This is a slight modification over the original weight function proposed in Ghosh (1996). The weight function is tuned through a suitable choice of the constant $C$. In our present work, we use this constant $C$ to modify the weights, leading to the diagonalisation of the least squares matrix (Konark \& Deshpande 2006). The immediate consequence of this is, all the 2-D least squares formulae reduce to equivalent 1-D form for all the derivatives. The details of this will be explained in section 5 .

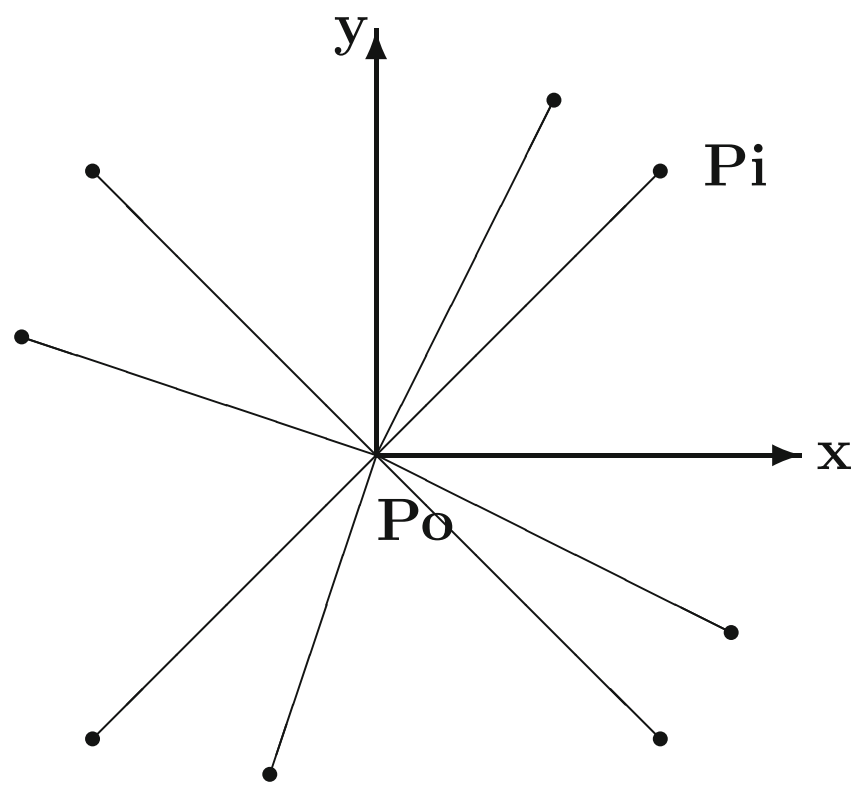

Figure 1. Sketch of typical point connectivity for LSKUM. 
Discretising the time derivative to first order and using least squares formulae to approximate the derivatives of the various split fluxes, an update scheme for 2-D Modified Moving KFVS split Euler equations can be written as,

$$
\begin{aligned}
& U^{n+1}=U^{n}-\Delta t {\left[\left\{\frac{\partial}{\partial x}\left(G X_{M}^{+}\right)\right\}_{N_{1}\left(P_{o}\right)}+\left\{\frac{\partial}{\partial x}\left(G X_{M}^{-}\right)\right\}_{N_{2}\left(P_{o}\right)}+\right.} \\
&\left.\left\{\frac{\partial}{\partial y}\left(G Y_{M}^{+}\right)\right\}_{N_{3}\left(P_{o}\right)}+\left\{\frac{\partial}{\partial y}\left(G Y_{M}^{-}\right)\right\}_{N_{4}\left(P_{o}\right)}\right]^{n} .
\end{aligned}
$$

In the above update formula, we choose a suitable subset of points in the connectivity (referred to as sub stencil) to ensure that the signal (Ramesh 2001) propagation property is not violated. The subscripts to the various flux derivative approximations indicate the sub stencil chosen from the full connectivity set. Figure 2 shows the various sub stencils chosen in the least squares approximation to the derivatives. For computing the x-split derivatives, the stencil is divided into two sub stencils, $N_{1}\left(P_{o}\right)$ and $N_{2}\left(P_{o}\right)$ as shown in the figure. Sub stencil $N_{1}\left(P_{o}\right)$ is used for the computation of positive x-split fluxes and sub stencil $N_{2}\left(P_{o}\right)$ is used for computing the negative X-split fluxes. Similarly, sub stencils $N_{3}\left(P_{o}\right)$ and $N_{4}\left(P_{o}\right)$ is used for computing the positive and negative y-split fluxes, respectively. The dissipation control parameters $\phi_{1}$ and $\phi_{2}$ are chosen as $\phi_{1}=\phi_{2}=\Delta r^{p}$, where $0<p<1$ and $\Delta r$ is the distance between point $P_{o}$ and any point in the connectivity. We usually choose the closest point. This approach helps us in getting a higher order accurate estimate of the derivatives even while using a first order type of least squares formulae. The proof of this fact has been given in Ramesh \& Deshpande (2007). Having
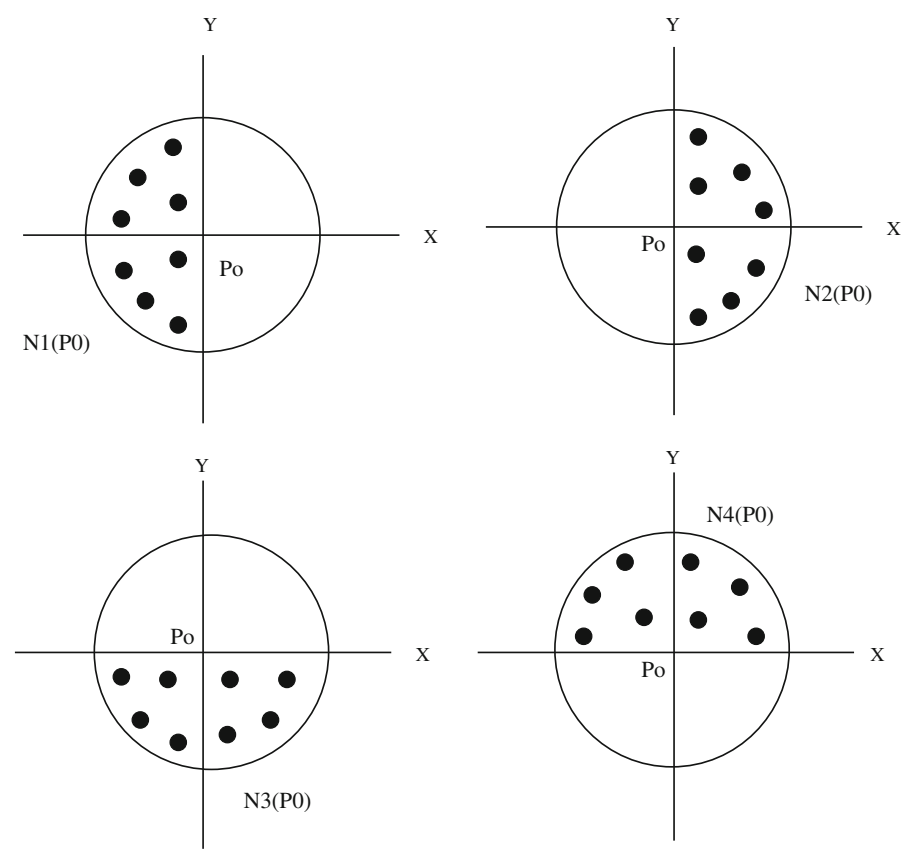

Figure 2. Stencil splitting for LSKUM. 
briefly described, the state update of an interior point we now proceed to describe the treatment of boundary condition on moving wall.

\section{Kinetic treatment of boundary condition for moving solid wall}

Consider a boundary node $b$ lying on a moving surface $S$ as shown in figure 3. Let $w_{1}$ and $w_{2}$ be the components of the node velocity along the tangential and the normal direction $\mathrm{X}-\mathrm{Y}$.

Following the specular reflection principle (Mandal \& Deshpande 1994), the velocity distribution function $f_{b}$ at $b$ is the union of two half Maxwellians $F_{I}$ and $F_{R}$ corresponding to the incident and reflected particles, that is,

$$
f_{b}=F_{I} \cup F_{R} .
$$

The incident part corresponds to the particles with the relative velocity normal to the wall $\bar{v}_{2}<0$. The reflected part is constructed from the incident part as shown below.

$$
\begin{gathered}
F_{I}=F_{I}\left(\bar{v}_{1}, \bar{v}_{2}\right) \quad \text { for } \quad \bar{v}_{2}<0, \\
F_{R}=F_{I}\left(\bar{v}_{1},-\bar{v}_{2}\right) \text { for } \quad \bar{v}_{2}>0 .
\end{gathered}
$$

Using the MCIR splitting for $\bar{v}_{1}$ the update formula for a solid boundary point $b$ is then given by

$$
\left\langle\Psi, f_{b}^{n+1}\right\rangle \equiv U_{b}^{n+1} \equiv\left\langle\Psi, F_{I}^{n+1} \cup F_{R}^{n+1}\right\rangle
$$

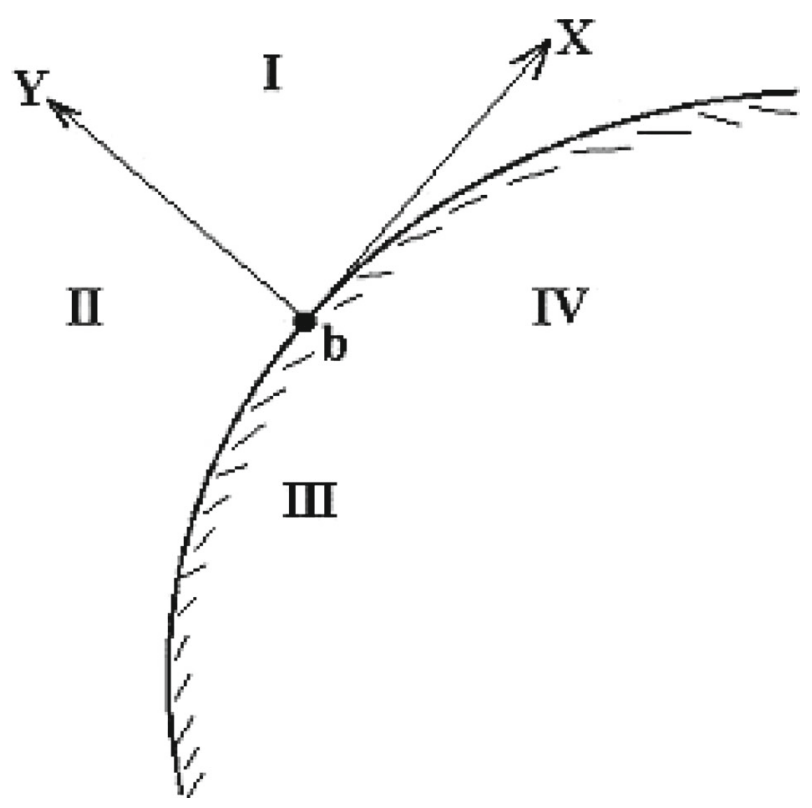

Figure 3. A typical 2-D boundary. 
where

$$
\begin{aligned}
& F_{I}^{n+1}=F_{I}^{n}-\Delta t\left(\frac{\bar{v}_{1}+\phi_{1}\left|\bar{v}_{1}\right|}{2} \frac{\partial F}{\partial x}+\frac{\bar{v}_{1}-\phi_{1}\left|\bar{v}_{1}\right|}{2} \frac{\partial F}{\partial x}+\bar{v}_{2} \frac{\partial F}{\partial y}\right)^{n} \text { for } \quad \bar{v}_{2}<0 . \\
& F_{R}^{n+1}=F_{I}^{n+1}\left(\bar{v}_{1},-\bar{v}_{2}\right) \text { for } \bar{v}_{2}>0 .
\end{aligned}
$$

The details of the various modified split moving fluxes which arise in this boundary treatment are given in Ramesh \& Deshpande (2007).

\section{Kinetic treatment of inflow, outflow and periodic boundary condition}

The treatment of the boundary condition for a node on these boundaries is very much similar to the kinetic outer boundary condition (Ramesh 2001). The velocity distribution $f_{b}$ at any point $b$ on these boundaries is constructed as the union of two half Maxwellians $F_{\text {in }}$ and $F_{\text {out }}$ corresponding to incoming and outgoing particles. The velocity distribution $F_{\text {in }}$ corresponding to the incoming particles is constructed from the inlet/exit conditions specified. In case of periodic boundary there are two curves one at the top and another at the bottom as shown in figure 4 . For every point on this curve, the velocity distribution corresponding to the incoming particles is constructed from the conditions at a similar point on the other part of the periodic boundary. The velocity distribution function $F_{\text {out }}$ for the outgoing particles is obtained from the interior points. The velocity distribution function $f_{b}$ at $b$ is then given by,

$$
f_{b}^{n+1}=F_{\text {in }}^{n+1} \cup F_{\text {out }}^{n+1} .
$$
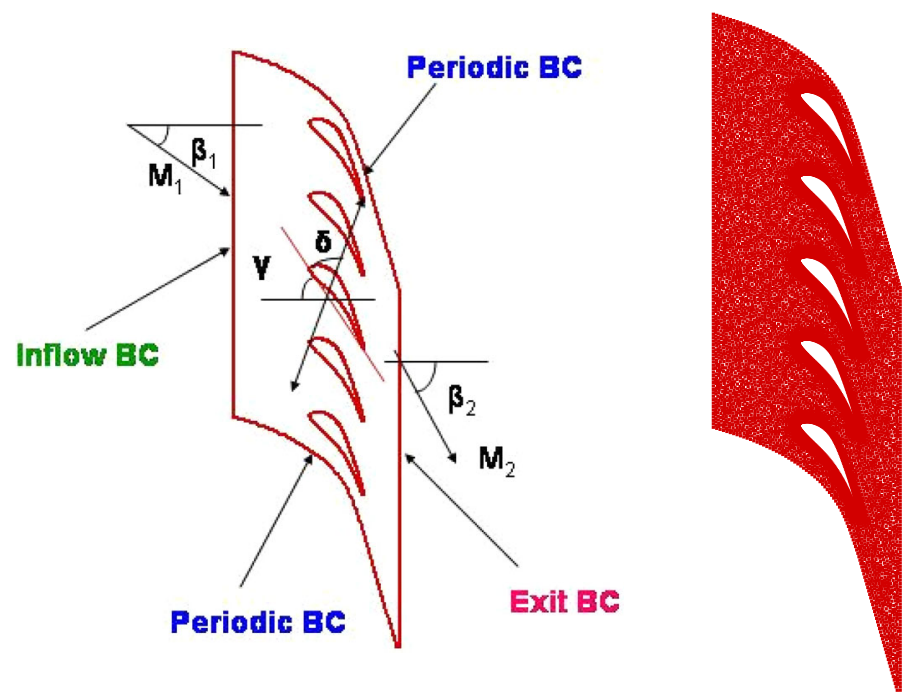

Figure 4. (1) Problem definition and (r) points distribution for the STCF4 cascade configuration. 
Using the MCIR splitting for $\bar{v}_{1}, F_{\text {in }}$ and $F_{\text {out }}$ can be written as:

$$
\begin{aligned}
F_{i n}^{n+1} & =F_{s} \quad \text { for } \quad \bar{v}_{2}<0, \\
& =\frac{\rho_{s}}{I_{0_{s}}} \frac{\beta_{s}}{\pi} \exp \left[-\beta\left(v_{1}-u_{1_{s}}\right)^{2}-\beta\left(v_{2}-u_{2_{s}}\right)^{2}-I / I_{0}\right], \\
F_{\text {out }}^{n+1} & =F_{\text {out }}^{n}-\Delta t\left(\frac{\bar{v}_{1}+\phi_{1}\left|\bar{v}_{1}\right|}{2} \frac{\partial F}{\partial x}+\frac{\bar{v}_{1}-\phi_{1}\left|\bar{v}_{1}\right|}{2} \frac{\partial F}{\partial x}+\bar{v}_{2} \frac{\partial F}{\partial y}\right)^{n} \text { for } \bar{v}_{2}>0 .
\end{aligned}
$$

The conditions specified at inlet/exit/periodic boundaries is indicated by the subscript $s$. Taking $\Psi$ moments of the Eq. (20) we get the update formula for all these boundary points. The details of the formula can be seen in Ramesh \& Deshpande (2007).

\section{Weighted LSKUM_MN using Eigenvector basis}

Consider the first order weighted least squares approximation (Ghosh \& Deshpande 1995) given by the following equation,

$$
A(w) \Delta F=b(w)
$$

where

$$
A(w)=\left[\begin{array}{ll}
\sum w_{i} \Delta x_{i}{ }^{2} & \sum w_{i} \Delta x_{i} \Delta y_{i} \\
\sum w_{i} \Delta x_{i} \Delta y_{i} & \sum w_{i} \Delta y_{i}^{2}
\end{array}\right], \Delta F=\left[\begin{array}{c}
F_{x} \\
F_{y}
\end{array}\right], b(w)=\left[\begin{array}{c}
\sum w_{i} \Delta x_{i} \Delta F_{i} \\
\sum w_{i} \Delta y_{i} \Delta F_{i}
\end{array}\right],
$$

where $w_{i}$ is the weight associated with each node $i$ in the connectivity. The weights in the matrix $A(w)$ can be suitably (Konark \& Deshpande 2006) chosen such that $A(w)$ gets diagonalised. Let us see how we can apply this to Euler equations.

Consider the 2-D KFVS MCIR split Euler equations for moving nodes,

$$
\left(\frac{d U}{d t}\right)_{m o v}+\frac{\partial}{\partial x}\left(G X_{M}^{+}\right)+\frac{\partial}{\partial x}\left(G X_{M}^{-}\right)+\frac{\partial}{\partial y}\left(G Y_{M}^{+}\right)+\frac{\partial}{\partial y}\left(G Y_{M}^{-}\right)=0 .
$$

In order to diagonalize the least squares matrix corresponding to each of the derivative of the split flux, we should clearly recognise that a different set of weights are required. This also implies that the set of weights for each matrix leads to eigendirections to coincide with the $\mathrm{X}-\mathrm{Y}$ directions. This approach is referred to as the Weighted Least Squares using EigenVector basis.

Separate set of weights are associated for points in each of the subsets $N_{1}, N_{2}, N_{3}$ and $N_{4}$ corresponding to the evaluation of the various split flux derivatives as indicated in the previous sections. A detailed derivation of the weights for various subsets are given in Ramesh \& Deshpande (2007).

Time accurate solutions with explict codes using global time stepping will take enormous amount of computational time. In order to reduce this, we have used the well-established dual time stepping approach, which helps in much faster convergence. The details of this implementation can be found in Ramesh \& Deshpande (2007). 


\section{Results and discussion}

A set of cascade standard configurations, with emphasis on the unsteady aerodynamics, is available in Bolcs \& Fransson (1986). A further updated report is available in Fransson \& Verdon (1991). These reports contain comprehensive set of experimental data for various test flow conditions related to the aeroelastic problem. All the results are categorised into 11 standard configurations. A detailed presentation for each test case can be found in these references. The test case we have chosen is the 4th standard aeroelastic test case. This test case refers to an annular turbine cascade in which the blades oscillate sinusoidally in the first bending mode. The same oscillation amplitude and frequency are imposed for all the blades whereas the IBPA (Inter Blade Phase Angle) is a variable. A multipassage computational domain is employed, using a number of blade passages, $n_{p}\left(n_{p}>1\right)$ given by

$$
n_{p}=\frac{360^{\circ} z}{|\mathrm{IBPA}|}+1, \quad \mathrm{IBPA} \neq 0,
$$

$z$ is the minimum integer which leads to an integer value for $n_{p}$. This approach allows us to use periodic boundary conditions even for cases with IBPA $\neq 0$. This also avoids the direct store (Erdos \& Alzner 1977) method used with single passage. One of the main difficulties for turbomachinery flutter calculations is the grid generation as well as grid movement strategy required in an environment where multiple blades are in oscillation. Usual approach is to use multiblock overlapped meshes with inter block boundary conditions imposed by bilinear interpolation (Cinnella et al 2004). However, with our present TKFMG code we do not face any such difficulties because the mesh-free method has the capability to just operate on any arbitrary distribution of points with random node velocities. In our present work, the nodes lying exactly on the blades are moved according to the motion prescribed for the blades. The nodes on the inflow, exit and the periodic boundaries are held fixed. For the rest of the nodes the motion exponentially decreases from the blades towards the fixed boundaries. As the blades oscillate, the points in the domain also move, hence the connectivity continuously changes. In our code we have an inbuilt connectivity generation preprocessor which updates the connectivity continuously as the points move.

The inlet flow Mach number $M_{1}$ is 0.28 , with exit isentropic Mach number $M_{2}=0.9$ figure 4 shows the details of the various inflow/exit and periodic boundary conditions. The inlet flow angle is $\beta_{1}=45^{\circ}$ inclined downward with respect to the axial direction and the corresponding exit flow angle $\beta_{2}=72^{\circ}$. The chordal stagger angle $\gamma=56.6^{\circ}$ and the direction of vibration with respect to the chord line is given by $\delta=60.4^{\circ}$. This corresponds to the harmonic motion of the blades in first bending mode. The amplitude of vibration is $0.33 \%$ of the chord length and reduced frequency $k$, based on the half-chord length and the outlet conditions, is equal to 0.107 .

The computational point cloud consists of a total number 16,976 nodes with 306 nodes on each blade. This represents fairly a fine point cloud. This size of the point cloud has been arrived at based on the experience gained with this method for various applications. As already mentioned in the previous section, we use Eigen direction based weights for the least squares approximation of derivatives. For every point in the connectivity, the weight chosen is of the form $w_{i}=C_{i} / d_{i}^{2 n}$, where $i$ represents any point in the connectivity and $d$ represents the distance between $i$ and the node at which update of solution is carried out. In this particular choice of weight function, the constant $C_{i}$ is suitably chosen to diagonalise the least squares matrix. The factor $1 / d_{i}^{2 n}$ ensures the local property of the derivative by giving more weight to closer points in the connectivity. Apart from these weights, at every node a dissipation control parameter $\phi$ defined as $\phi=(\Delta r)^{p}$ 
is used, where $\Delta r$ is the distance between the node and its closest point in the connectivity. This parameter is quite different from the weights mentioned previously. The dissipation control parameter is used to modify the formal order of accuracy of the scheme while the Eigen direction based weights are used for derivative approximation. In the present computations, we use $n=3$ and $p=0.3$. The steady state solution is used as the initial condition for unsteady computations. For steady flows, the solution is marched in time using a four-stage Runge-Kutta scheme. For unsteady flow computations, the time derivative is discretized using a two level second-order accurate Crank-Nicholson method and the dual time stepping procedure is used. We have used sixty physical time steps to compute each cycle. For each physical time step, in the inner loop we use 1000 iterations to converge in the pseudo local time steps. All the unsteady computations are typically carried out for about five cycles in which the periodic behaviour of the flow is captured. Figure 4 shows the points distribution used for the computations. Figure 5 shows the steady state pressure contours for single blade computation and for the cascade with five blades. It can be observed that we have captured essentially all the features exactly same in both the cases. This can be further verified in figure 6 which shows the isentropic Mach number distribution for single blade as well as cascade computations. Exactly similar solutions for all the blades can be obtained only when the periodic boundary condition is accurately implemented. It is important that we ensure this symmetric steady solution, because this solution in turn is used as an initial condition for the unsteady flow computations.

Unsteady computations have been done for various IBPA's. Unsteady $C_{p}(t)$ is defined as (Fransson \& Verdon 1991)

$$
C_{P}(t)=\frac{p(t)}{\bar{h}_{\max }\left(p_{o}-p_{\infty}\right)_{\text {inlet }}}
$$

where $p_{o}$ is the stagnation pressure at the inlet, $p_{\infty}$ is the static pressure at inlet, $\bar{h}_{\max }=$ $h_{\max } / C, C$ is the chord length of the blade and $h_{\max }$ is the amplitude of vibration. Unsteady $C_{p}(t)$ is expressed in a fourier series, as

$$
C_{P}(t)=C_{P_{0}}+C_{P_{1}} \sin \left(w t+\epsilon_{1}\right)+C_{P_{2}} \sin \left(2 w t+\epsilon_{2}\right)+\cdots,
$$
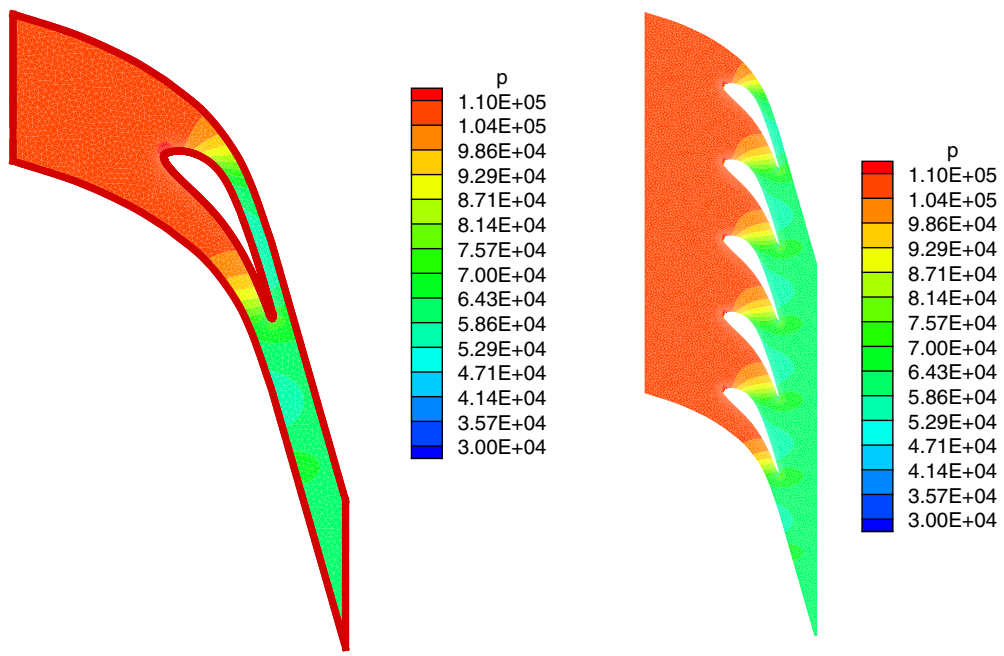

Figure 5. Steady state pressure contours - (1) single blade, (r) cascade. 


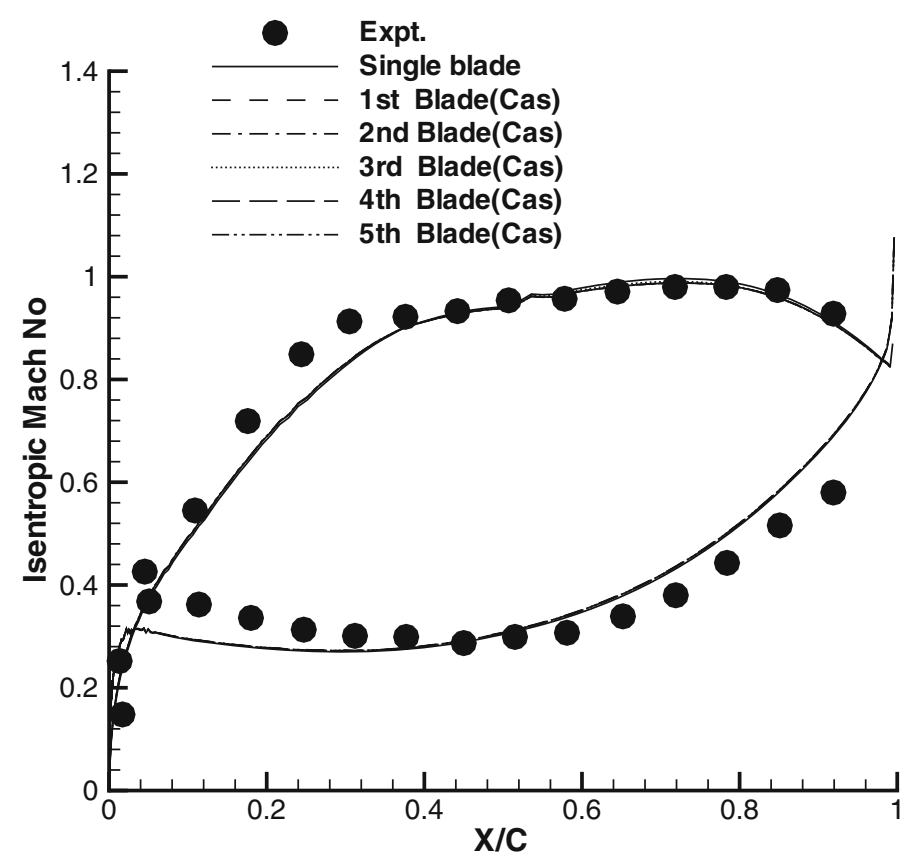

Figure 6. Isentropic Mach number plot.

where $w=2 \pi f, f$ is the frequency of the vibration in the first bending mode, $C_{P_{i}}$ is the amplitude of the $i$ th component and $\epsilon_{i}$ is the phase shift. The experimental results are available in terms of the amplitude and phase of the unsteady $C_{P}$ for the first hormonic. In our present work, we have compared our predictions with the experiments in terms of these two quantities. However amplitude and phase of the higher hormonics are available from the computations. But these are not plotted since in general the amplitudes of the higher hormonics are very small compared to the fundamental. Comparison of the phase assumes significance in the view that it is crucial in the correct prediction of the flutter instability. In the present work, we have chosen IBPA's corresponding to $-90^{\circ}, 90^{\circ}, 180^{\circ}$ and $0^{\circ}$. These cases correspond to the test cases numbers 3 , 6,7 and 8 , respectively for the fourth standard configuration. It is for these test cases extensive computations have been reported in the literature.

Figure 7 shows a comparison of the computations with the experiment for the first harmonic of the unsteady $C_{p}$ for IBPA $=90^{\circ}$. It can be noticed that the calculation shows higher amplitudes over the front half chord. However, the trend is predicted well. This behaviour has been reported by many previous computations. In the figure we also show a comparison with the results of Mani \& Feng (2001) obtained by finite volume Euler computations. Very similar behaviour is also reported in Ji \& Liu (1999) using Euler and N-S computations. As already pointed out by Mani \& Feng (2001) for this test case higher prediction of amplitudes have been mentioned in the original reports (Bolcs \& Fransson 1986; Fransson \& Verdon 1991). The exact reason is not known, however report by Fransson (Fransson \& Verdon 1991) suggests the discrepancy could be due to uncertainty of the inflow angle, leakage flow and boundary layer growth in the test facility. The mismatch is in general more pronounced for the transonic flow conditions. In fact, the computations by Ji \& Liu (1999), Mani \& Feng (2001) uses a stream-tube contraction model in the calculations to compensate for leakage flow and boundary layer growth in the test 

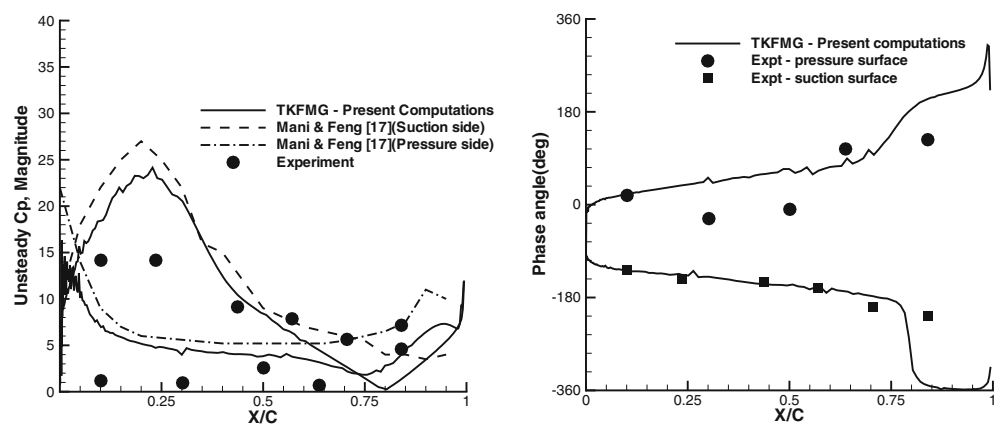

Figure 7. First harmonic of unsteady pressure - IBPA $=90^{\circ}$, (l) Amplitude, (r) Phase.

facility. In our present work we do not use any such corrections but still we are able to predict the behaviour comparable to other methods as mentioned earlier. Euler and $\mathrm{N}-\mathrm{S}$ computations by Napalitano (Cinnella et al 2004) is another example where flutter computations for this test case have been carried out without any such corrections for the stream-tube contractions. Even in this work the amplitudes are not consistently predicted for all the IBPA's. Figure 7 shows the phase plot for IBPA $=90^{\circ}$. The discrepancies in phase is much less, and this ensures the correct stability range prediction. Similar amplitude and phase plots can be seen in figures 8 and 9 for IBPA $=180^{\circ}$ and $-90^{\circ}$, respectively.

Finally, the most important parameter needed to predict flutter is the computation of the aerodynamic damping from the unsteady data. This represents the work done per cycle on the blade by the aerodynamic forces. A negative value of this coefficient represents the energy transfer from the flow to the blades indicating flutter. Net energy transfer to the blade in one period is calculated as:

$$
W_{A}=\oint \int_{0}^{T}-p(x, t) \underset{\sim}{n} d s \cdot\left(i \frac{d \mathrm{X}_{B}}{d t}+j \frac{d \mathrm{Y}_{B}}{d t}\right) d t,
$$

where $p(x, t)$, is the pressure at any point on the blade, $\mathrm{X}_{B}, \mathrm{Y}_{B}$, co-ordinates of the point on the blade non-dimensionalised with chord length, $n$, normal at the point on the blade, $T$ is the time period of each oscillation, and $s$ arc length non-dimensionalised with chord length.
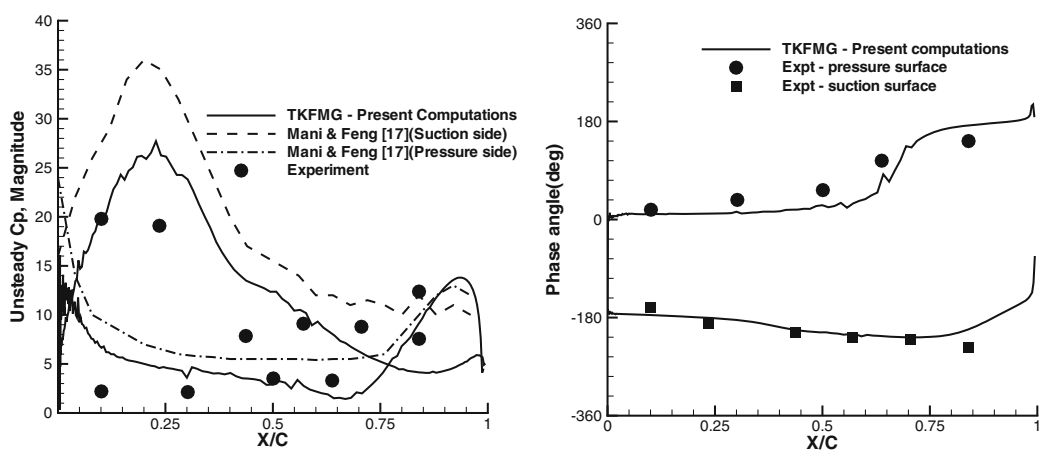

Figure 8. First harmonic of unsteady pressure - IBPA $=180^{\circ}$, (l) Amplitude, (r) Phase. 

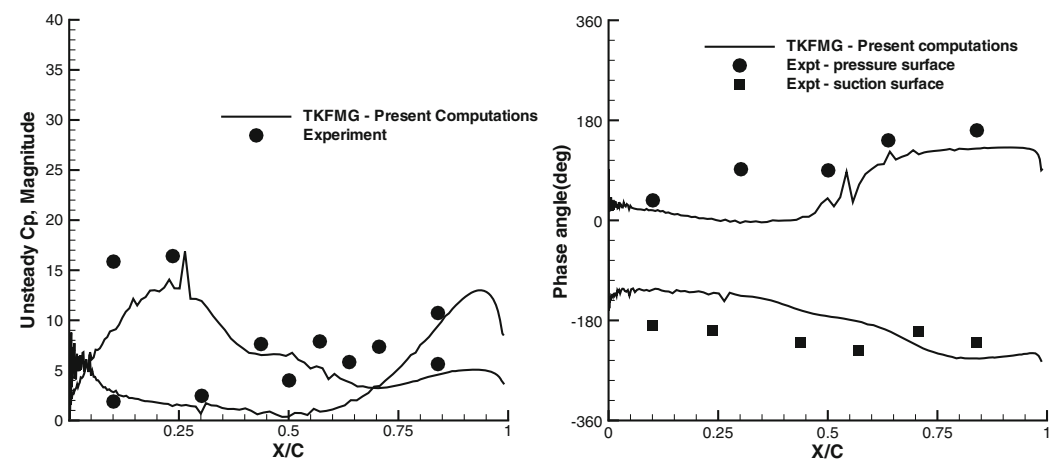

Figure 9. First harmonic of unsteady pressure - IBPA $=-90^{\circ}$, (l) Amplitude, (r) Phase.

Aerodynamic damping coefficient is defined as

$$
\Xi=\frac{-W_{A}}{\left(p_{o}-p\right)_{\text {inlet }} \bar{h}_{\text {max }}^{2}},
$$

where $\left(p_{o}-p\right)$, is the compressible dynamic pressure at the upstream.

Figure 10 shows the plot for aerodynamic damping coefficient for various IBPA's. It can be seen that our TKFMG code clearly shows that flutter is present for IBPA $=-90^{\circ}$. Also in the figure we have shown the results of Euler computations by Ji \& Liu (1999), Gruber \& Cartsens (1998). It can be observed that the trend is captured correctly, that is, regions of positive and

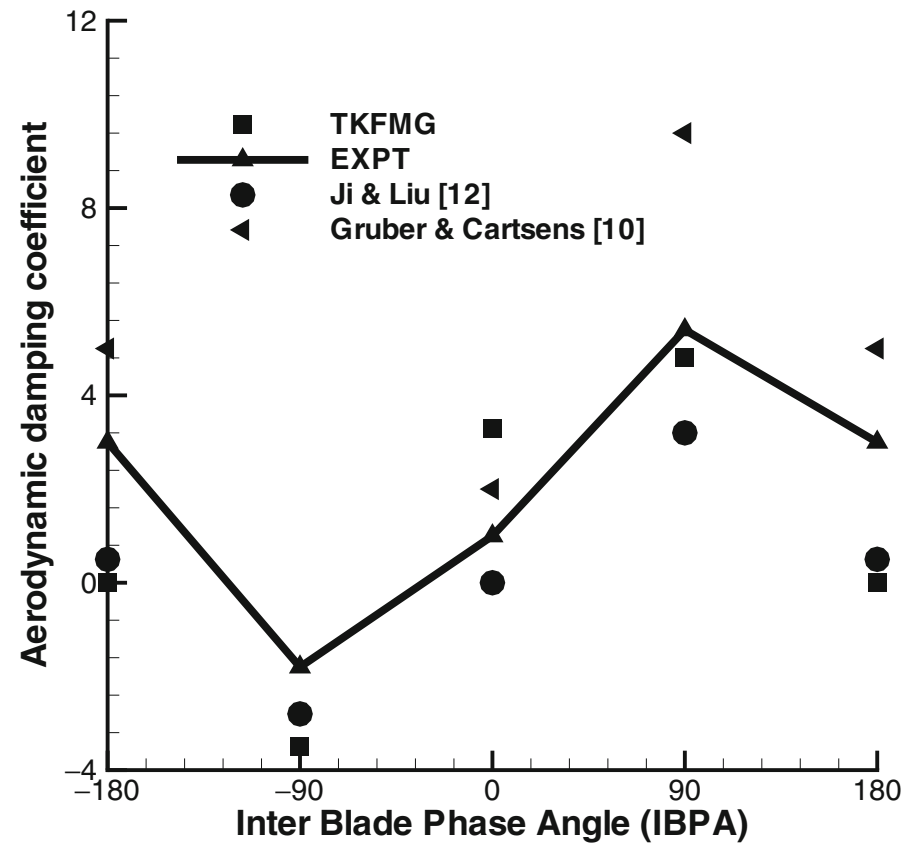

Figure 10. Aerodynamic damping. 
negative aerodynamic damping given by computations are in agreement with those observed by experiments.

\section{Conclusions}

In this work, we have demonstrated for the first time the power of the mesh-free method to compute unsteady flow in oscillating turbine cascade. The present method naturally blends to both moving as well as static nodes without the need for any sort of complex interpolation as needed by all other conventional solvers. We feel this approach has a tremendous potential to handle challenging applications in turbomachines such as multi-stage computations with rotor/stator interactions, tip flow analysis with casing, etc. For the 4th standard aeroelastic test case, we have obtained results comparing well with the experiments for the unsteady pressure coefficients. Finally, we have been able to predict the flutter with our TKFMG code for the 4th standard aeroelastic test case by computing the aerodynamic damping coefficient.

\section{References}

Bolcs A and Fransson T H 1986 Aeroelasticity in Turbomachines, Comparison of Theoretical and Experimental Cascade Results, communication du Laboratoire de Thermique Appliquee et de Turbomachines, No. 13, EPFL, Lausanne, Switzerland

Carta F O 1967 Coupled Blade-Disc-Shroud flutter Instabilities in Turbo-jet engine Rotors. J. Eng. Power 89(3): 419-426

Cinnella P, De Palma P, Pascazio G and Napolitano M 2004 A numerical method for turbomachinery aeroelaticity. Trans. ASME 126: 310-316

Deshpande S M 1986 Kinetic theory based new upwind methods for inviscid compressible flows, AIAA Paper 86-0275

Erdos J I and Alzner E 1977 Numerical solution of periodic transonic flow through a fan stage. AIAA J. 15: $1559-1568$

Fransson T H and Verdon J M 1991 Updated report on standard configurations for unsteady flow through vibrating axial-flow turbomachine-cascades, http://www.egi.kth.se

Ghosh A K and Deshpande S M 1995 Least squares kinetic upwind method for inviscid compressible flows, AIAA Paper 95-1735

Ghosh A K 1996 Robust least squares kinetic upwind method for inviscid compressible flows, Ph.D. Thesis, Indian Institute of Science, Bangalore

Gruber B and Cartsens V 1998 Computation of unsteady transonic flow in harmonically oscillating turbine cascades taking into account viscous effects. ASME J. Turbomach. 120: 104-120

Hong Luo, Joseph D Baum and Rainald Löhner 2001 An accurate, fast, matrix-free implicit method for computing unsteady flows on unstructured grids. Comput. Fluids 30: 137-159

Ji S and Liu F 1999 Flutter Computation of Turbomachinery Cascades Using a Parallel Unsteady NavierStokes Code. AIAA J. 37(3): 320-327

Konark Arora and Deshpande S M 2006 Weighted Least Squares Kinetic Upwind method using Eigenvector basis, $\mathrm{PhD}$ Thesis, Indian Institute of Science, Bangalore

Krishnamurthy R, Sarma B S and Deshpande S M 2004 Kinetic scheme for computational aeroelastic analysis of 2-D airfoils in transonic flows. 34th AIAA Fluid Dynamics Conference and Exhibit, 28 JuneJuly 2004, Portland, Oregon, 2004-2236

Mahendra A K 2003 Application of least squares kinetic upwind method to strongly rotating viscous flows, MSc (Engg) thesis, Indian Institute of Science, Bangalore

Mandal J C and Deshpande S M 1994 Kinetic flux vector splitting for Euler equations. Comput. Fluids 23: $447-478$ 
Mani Sadeghi and Feng Liu 2001 Computation of Mistuning Effects on Cascade Flutter. AIAA J. 39(1): 22-28

Ramesh V 2001 Least Squares Grid Free Kinetic Upwind Method, PhD thesis, Indian Institute of Science, Bangalore

Ramesh V and Deshpande S M 2001 Least Squares Kinetic Upwind Method on moving grids for unsteady Euler computations. Comput. Fluids J. 30(5): 621-641

Ramesh V and Deshpande S M 2004a Low dissipation grid free upwind kinetic scheme with modified CIR splitting, Fluid Mechanics Report 2004 FM 20, Centre of Excellence in Aerospace CFD, Dept. of Aero. Engg., Indian Institute of Science, Bangalore

Ramesh V and Deshpande S M 2004b Least squares kinetic upwind method with modified CIR splitting, Proc. 7th Annual CFD symp., August 11-12 2004, National Aerospace Laboratories, Bangalore, India

Ramesh V and Deshpande S M 2007 Unsteady flow computations for flow past multiple moving boundaries using LSKUM. Comput. Fluids J. 36: 1592-1608 